\title{
Upregulation of USP11 promotes epithelial-to-mesenchymal transition by deubiquitinating Snail in ovarian cancer
}

\author{
WEIWEI WANG ${ }^{1}$, JING WANG $^{1}$, HUA YAN $^{1}$, KAI ZHANG $^{2}$ and YANG LIU ${ }^{1}$ \\ Departments of ${ }^{1}$ Obstetrics and ${ }^{2}$ Orthopaedics, Linyi Central Hospital, Linyi, Shandong 276400, P.R. China
}

Received December 7, 2017; Accepted July 26, 2018

DOI: $10.3892 / o r .2018 .6924$

\begin{abstract}
Ubiquitin specific peptidase 11 (USP11) is a deubiquitinating enzyme (DUB) and has been observed to promote mammary tumor initiation and progression. Several reports have shown that USP11 plays an important role in cancer development. Previous research has shown that USP11 is upregulated in breast cancer, but the function of USP11 in ovarian cancer has not been determined to date. In the present study, we observed that USP11 was upregulated in ovarian cancer tissues and cell lines. To determine the role of USP11 in ovarian cancer, we performed several functional experiments, including Transwell migration and invasion analysis, in vitro and in vivo deubiquitination assays, colony formation assays, and CCK-8 assays. Notably, we observed that USP11 facilitated the epithelial-to-mesenchymal transition (EMT), which is an important process in cancer cell invasion and metastasis. Furthermore, we observed that USP11 regulated EMT through the regulation of Snail. Our results revealed that USP11 promoted EMT in ovarian cancer by deubiquitinating Snail, and USP11 may be a novel therapeutic target for ovarian cancer treatment.
\end{abstract}

\section{Introduction}

Deubiquitinating enzymes (DUBs) play a critical function through regulation of protein-protein interactions, protein subcellular localization and protein stability $(1,2)$. Many DUBs, such as USP11, USP22 and USP37 have been found to play important functions in several types of cancers (3-7).

Recently, several reports have found that USP11 plays an important role in modulating a number of signaling cascades, including NF- $\kappa \mathrm{B}$, TGF- $\beta$ and Notch signaling pathways, and the DNA damage response (5,8-11). USP11 exhibits differential functions through interacting with multiple substrates,

Correspondence to: Dr Yang Liu, Department of Obstetrics, Linyi Central Hospital, 17 Health Road, Linyi, Shangdong 276400, P.R. China

E-mail: o337c3d@163.com

Key words: USP11, epithelial-to-mesenchymal transition, Snail, metastasis, ovarian cancer such as promyelocytic leukemia protein (PML), BRCA2 and I $\kappa \mathrm{B} \alpha(5,8-11)$. For example, USP11 interacts with and deubiquitylates $\mathrm{I} \kappa \mathrm{B}$, thereby inhibiting $\mathrm{NF} \kappa \mathrm{B}$ activation (10). A recent study showed that USP11 is upregulated in breast cancer, and its abnormal accumulation predicts a poor patient prognosis (6). However, the function of USP11 in ovarian cancer remains unknown.

Cancer metastasis is one of the important causes of the poor outcomes of tumor patients and is a complex, multistep process (12). Increasing motility and invasiveness of cancer cells results in the initiation of metastasis. To increase their invasive abilities, cancer cells undergo physiological changes, including an epithelial-to-mesenchymal transition (EMT) (13). EMT is a complex process in which epithelial cells convert into a mesenchymal phenotype. In addition, EMT plays crucial functions in tumor progression and metastasis. Several signaling pathways, such as the tyrosine kinase receptors, Wnt/ $\beta$-catenin, TGF- $\beta$, PI3K/Akt pathways and Notch regulate and induce EMT. One characteristic of EMT is the downregulation of E-cadherin and upregulation of $\mathrm{N}$-cadherin. Moreover, there are multiple transcription factors that have been found to respond to EMT, including Slug, Twist and Snail (14-16).

In this study, we verified that USP11 was significantly upregulated in ovarian cancer tissues and cell lines. In addition, high expression of USP11 was closely correlated with tumor size, TNM stage, lymph node metastasis, and predicted a poor prognosis of ovarian cancer patients. USP11 is a deubiquitinase, and we found that it interacted with Snail, a key EMT-associated transcription factor, and its deubiquitinase activity was dependent on a stabilized Snail. Our research revealed the detailed mechanism of USP11 in ovarian cancer, and suggests that it may be a novel molecular therapy target for ovarian cancer.

\section{Materials and methods}

Tissue specimens. A total of 143 pairs of ovarian cancer tissues and adjacent normal tissues were used for RT-qPCR and western blotting analysis. These patients included 143 females aged between 25-84 years, with a mean of 40.3 years. None of the patients had received chemotherapy or radiotherapy before surgery. The specimens were immediately stored at $-80^{\circ} \mathrm{C}$ before using. The Ethical Committees of Linyi Central Hospital approved all human tissue experiments 
and all patients provided written informed consent prior to participation in the study.

Cell culture. Human ovarian cancer cell lines, OVCAR-3 and SKOV3 and human normal ovarian cell line IOSE80 were obtained from the National Infrastructure of Cell Line Resource (Beijing, China). All cells were cultured in HyClone $\mathrm{H}^{\mathrm{TM}}$ Dulbecco's modified Eagle's medium (DMEM; GE Healthcare Life Sciences, Logan, UT, USA) supplemented with $100 \mathrm{U} / \mathrm{ml}$ penicillin, $100 \mathrm{~g} / \mathrm{ml}$ streptomycin (Thermo Fisher Scientific, Inc., Waltham, MA, USA) and $10 \%$ fetal bovine serum (FBS; Thermo Fisher Scientific, Inc.) (17). All cells were cultured at $37^{\circ} \mathrm{C}$ in a humidified atmosphere containing $5 \% \mathrm{CO}_{2}$.

Celltransfection. The vector(pcDNA3.1) andpcDNA3.1-USP11 were purchased from Vigene Biosciences (Shandong, China), and small-interfering RNA (siRNA) targeting USP11 and scramble siRNA (SCR) were chemically synthesized by Shanghai GenePharma Co., Ltd. (Shanghai, China). The cells were grown to $70-80 \%$ confluence prior to transfection in 6 -well plates. For cell transfection, cells were transfected with $2.5 \mu \mathrm{g}$ plasmid or $50 \mathrm{nM}$ siRNA using Lipofectamine 2000 (Invitrogen; Thermo Fisher Scientific, Inc.) according to the manufacturer's protocol. After transfection for $48 \mathrm{~h}$, the expression of USP11 was determined by qRT-PCR. USP11 siRNA-1: 5'-UUAUCUCAUCUUGAAAGAGUG-3'; USP11 siRNA-2: 5'-UGUUGUUGUUCAUGACAUGCA-3'; scramble siRNA (SCR): 5'-GAACCGTGTCTTCCTCAGTATC-3'.

Western blotting. After transfection for $48 \mathrm{~h}$, cells were collected and washed with cold-PBS three times, and lysed with RIPA buffer (1\% NP-40, $50 \mathrm{mM}$ Tris $\mathrm{pH}$ 7.4, 0.1\% SDS, $1 \mathrm{mM}$ EDTA, $0.5 \%$ DOC and $50 \mathrm{mM} \mathrm{NaCl}$ ) containing the protease inhibitor mixture (Sigma-Aldrich; Merck KGaA, Darmstadt, Germany) at $4^{\circ} \mathrm{C}$ for $45 \mathrm{~min}$. Lysates were centrifuged at $13,000 \times \mathrm{g}$ for $15 \mathrm{~min}$ at $4^{\circ} \mathrm{C}$, and the supernatants were removed to a new tube. The protein concentration was measured by the Bradford assay (Thermo Fisher Scientific, Inc.). Proteins ( $40 \mu \mathrm{g}$ ) were separated by $10 \%$ SDS-PAGE, and then transferred to polyvinylidene fluoride membranes (PVDF; GE Healthcare, Marlborough, MA, USA). Subsequently, the membranes were blocked with $5 \%$ skimmed milk at room temperature for $1 \mathrm{~h}$, followed by incubation with specific antibodies at $4^{\circ} \mathrm{C}$ overnight. The antibodies were as followed: USP11 (1:1,000; cat. no. ab109232; Abcam, Cambridge, MA, USA), EMT Kit (1:1,000; cat. no. 9782; Cell Signaling Technology, Inc., Danvers, MA, USA), $\beta$-actin $(1: 4,000$; cat. no. ab8226; Abcam). The membranes were washed with PBST three times and incubated with HPR-conjugated second antibodies (1:5,000; cat. nos. ab6721 and and 97023; Abcam) at room temperature for $1 \mathrm{~h}$ and washed with PBST three times. Finally, the specific blots were detected using ECL Western Blotting Detection Reagents (GE Healthcare, Marlborough, MA, USA). The densitometry of blots were analysized using ImageJ software (version 4.7; National Institutes of Health, Bethesda, MD, USA).

Quantitative real-time PCR ( $R T$ - $q P C R)$. Total mRNA was extracted from cells or tissue samples using Invitrogen ${ }^{\mathrm{TM}}$ TRIzol reagent (Thermo Fisher Scientific, Inc.) according to the manufacturer's instructions, and reverse transcription reaction was conducted using the TransScript First-Strand cDNA Synthesis SuperMix Kit (Transgen Biotech, Co., Ltd., Beijing, China). Subsequently, $2 \mu \mathrm{g}$ cDNA samples were used to perform RT-qPCR using TransStart Top Green qPCR SuperMix (Transgen Biotech). Primers were designed as follows: E-cadherin forward, 5'-AAACATCATTGATGCAGA CC-3' and reverse, 5'-GATAGATTCTTGGGTTGGGTC-3'; fibronectinforward, 5'-AATGTGAACGACACATTCCA-3' and reverse, 5'-ACCACTTGAGCTTGGATAGG-3'; N-cadherin forward, 5'-CAAAGCCTGGAACATATGTG-3'; and reverse, 5'-GTTTGAAAGGCCATATGTGG-3'; Slug forward, 5'-ACA CATACAGTGATTATTTCCC-3' and reverse, 5'-ACTGTA GTCTTTCCTCTTCAT-3'; Snail forward, 5'-TCTAATCCA GAGTTTACCTTCCAG-3' and reverse, 5'-TGAAGTAGA GGAGAAGGACGA-3'; Twist forward, 5'-GTACATCGACTT CCTCTACC-3' and reverse, 5'-GAAACAATGACATCTAGG TCTC-3'; GAPDH forward, 5'-ATTTCCTGGTATGACAAC GA-3' and reverse, 5'-TTGATGGTACATGACAAGGTG-3'. GAPDH was used as an internal control. The RT-qPCR conditions were as follows: $4 \mathrm{~min}$ at $98^{\circ} \mathrm{C}$, denaturation at $98^{\circ} \mathrm{C}$ for $30 \mathrm{sec}$, annealing at $56^{\circ} \mathrm{C}$ for $30 \mathrm{sec}$ and extension at $72^{\circ} \mathrm{C}$ for $35 \mathrm{sec}$, performed for 32 cycles. The relative expression of gene was analyzed by the $2^{-\Delta \Delta \mathrm{C}(\mathrm{q})}$ method (18).

Co-immunoprecipitation (co-IP) assay. Cells were collected and washed with PBS three times, and the cells were subsequently lysed using $0.3 \%$ IP buffer (1 mM EDTA, $25 \mathrm{mM}$ Tris- $\mathrm{HCl} \mathrm{pH} 7.5,150 \mathrm{mM} \mathrm{NaCl}, 0.3 \% \mathrm{NP}-40)$ and protease inhibitor cocktail at $4^{\circ} \mathrm{C}$ for $30 \mathrm{~min}$. Subsequently, the cell lysates were centrifuged at $13,000 \mathrm{x}$ g for $30 \mathrm{~min}$ and the supernatants were collected. Next, $2 \mu \mathrm{g}$ USP11 antibody (cat. no. ab109232; Abcam) and $2 \mu \mathrm{g}$ IgG antibody (cat. no. ab190492; Abcam) were added into the cell lysates, respectively. After rotation at $4^{\circ} \mathrm{C}$ overnight, $50 \mu \mathrm{l}$ protein $\mathrm{A}$ beads (Thermo Fisher Scientific, Inc.) was added. After rotation at $4^{\circ} \mathrm{C}$ for $3 \mathrm{~h}$, the beads were collected by centrifugation at $800 \mathrm{x} \mathrm{g}$ for $5 \mathrm{~min}$. The beads were next washed with $0.1 \% \mathrm{IP}$ buffer (1 mM EDTA, 25 mM Tris- $\mathrm{HCl}$ pH 7.5, $150 \mathrm{mM} \mathrm{NaCl}$, $0.1 \%$ NP-40) and a protease inhibitor cocktail three times. After denaturing at $95^{\circ} \mathrm{C}$ for $10 \mathrm{~min}$, the supernatants were collected and used for western blot analysis.

Colony formation assay. After transfection for 48 h, 5,000 cells were placed in 6-well plates and cultured with serum-free media. Cells were incubated at $37^{\circ} \mathrm{C}$ in a humidified atmosphere containing $5 \% \mathrm{CO}_{2}$ for 10 days. Cells were washed with PBS three times and fixed with $10 \%$ methanol at room temperature for $15 \mathrm{~min}$, and stained with $1 \%$ crystal violet at room temperature for $10 \mathrm{~min}$, then the cells were washed with PBS. Finally, the number of colonies (cells $>50$ ) were counted under an Olympus CKX41 light microscope (Olympus Corp., Tokyo, Japan). Each experiment was repeated three times.

CCK-8 assay. After transfection for $48 \mathrm{~h}, \sim 2,000$ cells were placed in 96-well plates and cultured with $200 \mu 1$ media. Every $12 \mathrm{~h}, 20 \mu \mathrm{l}$ CCK-8 regent was added and incubated with cells at $37^{\circ} \mathrm{C}$ in a humidified atmosphere containing $5 \% \mathrm{CO}_{2}$ for $1 \mathrm{~h}$. The OD 450 was measured. The value of OD 450 indicated the cell viability. Each experiment was repeated three times. 
In vivo deubiquitination analysis. FLAG-Snail and HA-ubiquitin were transfected in OVCAR-3/siUSP11 cells. After transfection for $48 \mathrm{~h}$, cells were treated with $10 \mu \mathrm{M}$ MG132 for $6 \mathrm{~h}$ and then lysed with RIPA buffer. The ubiquitinated status of Snail was detected through western blotting analysis with the HA antibody.

In vitro deubiquitination analysis. Briefly, HA-ubiquitin and FLAG-Snail were co-expressed in OVCAR-3 cells. After transfection for $48 \mathrm{~h}$, cells were treated with $10 \mu \mathrm{M}$ MG132 for $6 \mathrm{~h}$ and FLAG antibody was used to isolated ubiquitinated Snail by IP analysis. In a parallel experiment, vector or Myc-wt-USP11 or Myc-USP11/C318S were expressed in OVCAR-3 cells, and purified by IP with anti-Myc Affinity Matrix (Roche Diagnostics, Mannheim, Germany). Next, Myc-USP11 was eluded with Myc peptide and dialyzed. Finally, the purified USP11 was incubated with ubiquitinated Snail in a deubiquitination reaction buffer [1 mM ATP, $5 \mathrm{mM}$ $\mathrm{MgCl}_{2}, 50 \mathrm{mM}$ HEPES (pH 7.5), 5\% glycerol, $1 \mathrm{mM}$ DTT and $100 \mathrm{mM} \mathrm{NaCl}$ ] at $30^{\circ} \mathrm{C}$ for $2 \mathrm{~h}$. The ubiquitinated status of Snail was determined by western blot analysis with an anti-HA antibody.

Transwell migration and invasion assays. After transfection of the SKOV3 cells with the plasmid or siRNA for $48 \mathrm{~h}$, $\sim 2 \times 10^{5}$ cells in serum-free medium were placed into a chamber with Matrigel (8- $\mu \mathrm{m}$ pore; BD Biosciences, San Diego, CA, USA) for invasion analysis or into the chamber without Matrigel (8- $\mu \mathrm{m}$ pore; BD Biosciences) for migration analysis. Then the chambers were put in the 24-well plates with DMEM/F-12 supplemented with $10 \%$ FBS. The cells were incubated at $37^{\circ} \mathrm{C}$ with $5 \% \mathrm{CO}_{2}$ for $18 \mathrm{~h}$. The cells on the topside were removed by a cotton swab and the cells on the underside were fixed with $10 \%$ methanol at room temperature for $15 \mathrm{~min}$, and stained with $1 \%$ crystal violet at room temperature for $10 \mathrm{~min}$, and then washed with water. Finally, the number of cells were counted under an Olympus CKX41 light microscope (Olympus Corp.). Each experiment was repeated three times.

Statistical analysis. Statistical analyses were performed using SPSS software 18.0 (SPSS, Inc., Chicago, IL, USA). All data are represented as the mean \pm SD. The significant associations between the expression of USP11 and clinicopathological parameters of ovarian cancer patients were determined by two-tailed $\chi^{2}$ tests. Survival curve analysis was assessed using the Kaplan-Meier method. Analysis of variance followed by Tukey's post hoc test was used to assess the differences between multiple groups. The differences between two groups were determined using two-tailed Student's t-test. $\mathrm{P}<0.05$ was considered to be a statistically significant difference.

\section{Results}

Association between USP11 expression and clinicopathological variables in the ovarian cancer cases. Previous research has found that USP11 is upregulated in breast cancer $(6,19)$. To verify the expression of USP11 in ovarian cancer, we first used RT-qPCR and western blot analysis to analyze the expression of USP11 in 143 pairs of ovarian cancer tissues from patients who received tumor resection at the Linyi Central Hospital
Table I. Relationship between the expression of USP11 and the clinicopathological features of the ovarian cancer cases $(n=143)$.

\begin{tabular}{|c|c|c|c|c|}
\hline \multirow[b]{2}{*}{ Variables } & \multirow[b]{2}{*}{$\begin{array}{l}\text { No. of } \\
\text { cases }\end{array}$} & \multicolumn{2}{|c|}{$\begin{array}{l}\text { USP11 protein } \\
\text { expression }\end{array}$} & \multirow[b]{2}{*}{ P-value } \\
\hline & & $\begin{array}{c}\text { Low } \\
(n=56)\end{array}$ & $\begin{array}{l}\text { High } \\
(n=87)\end{array}$ & \\
\hline Age (years) & & & & 0.367 \\
\hline$<40$ & 68 & 24 & 44 & \\
\hline$\geq 40$ & 75 & 32 & 43 & \\
\hline $\begin{array}{l}\text { Tumor size }(\mathrm{cm}) \\
\text { (diameter) }\end{array}$ & & & & 0.002 \\
\hline Small $(\leq 3)$ & 69 & 36 & 33 & \\
\hline Large $(\geq 3)$ & 74 & 20 & 54 & \\
\hline \multicolumn{5}{|l|}{ TNM stage } \\
\hline I-II & 76 & 36 & 40 & 0.032 \\
\hline III-IV & 67 & 20 & 47 & \\
\hline pT status & & & & 0.014 \\
\hline pT1 & 71 & 35 & 36 & \\
\hline pT2-4 & 72 & 21 & 51 & \\
\hline pN status & & & & 0.011 \\
\hline pN0 & 63 & 32 & 31 & \\
\hline pN1-2 & 80 & 24 & 56 & \\
\hline Metastasis & & & & 0.010 \\
\hline Yes & 65 & 18 & 47 & \\
\hline No & 78 & 38 & 40 & \\
\hline
\end{tabular}

The mean value of USP11 mRNA content in tumor cells was set as the standard. High expression was more than the mean value, low expression was less than the mean value.

during May 2013 and October 2016, and their paired adjacent normal tissues were used as the control group. As shown in Fig. 1A, we found that the mRNA and protein levels of USP11 were clearly higher in tumor tissues than that in adjacent normal tissues. Meanwhile, we next analyzed the association between the expression of USP11 and the clinico-pathological features of the ovarian cancer patients. The results demonstrated that higher expression of USP11 was significantly associated with tumor size, TNM stage, and the presence of lymph node metastasis (Table I). However, no significant associations were observed between the USP11 expression level and patient age (Table I). Subsequently, we analyzed the correlation between the expression of USP11 and the survival curve of the ovarian cancer patients. As shown in Fig. 1B, high expression of USP11 predicted a poor prognosis of the patients. To investigate the function of USP11 in ovarian cancer, we next detected the expression of USP11 in ovarian cancer cell lines, including OVCAR-3 and SKOV3, and the human normal ovarian cell line IOSE80 was used as a control. We found that USP11 was significantly upregulated in OVCAR-3 and SKOV3 cells compared to that in the IOSE80 cells (Fig. 1C). Together, our findings showed that USP11 is upregulated in ovarian cancer. 

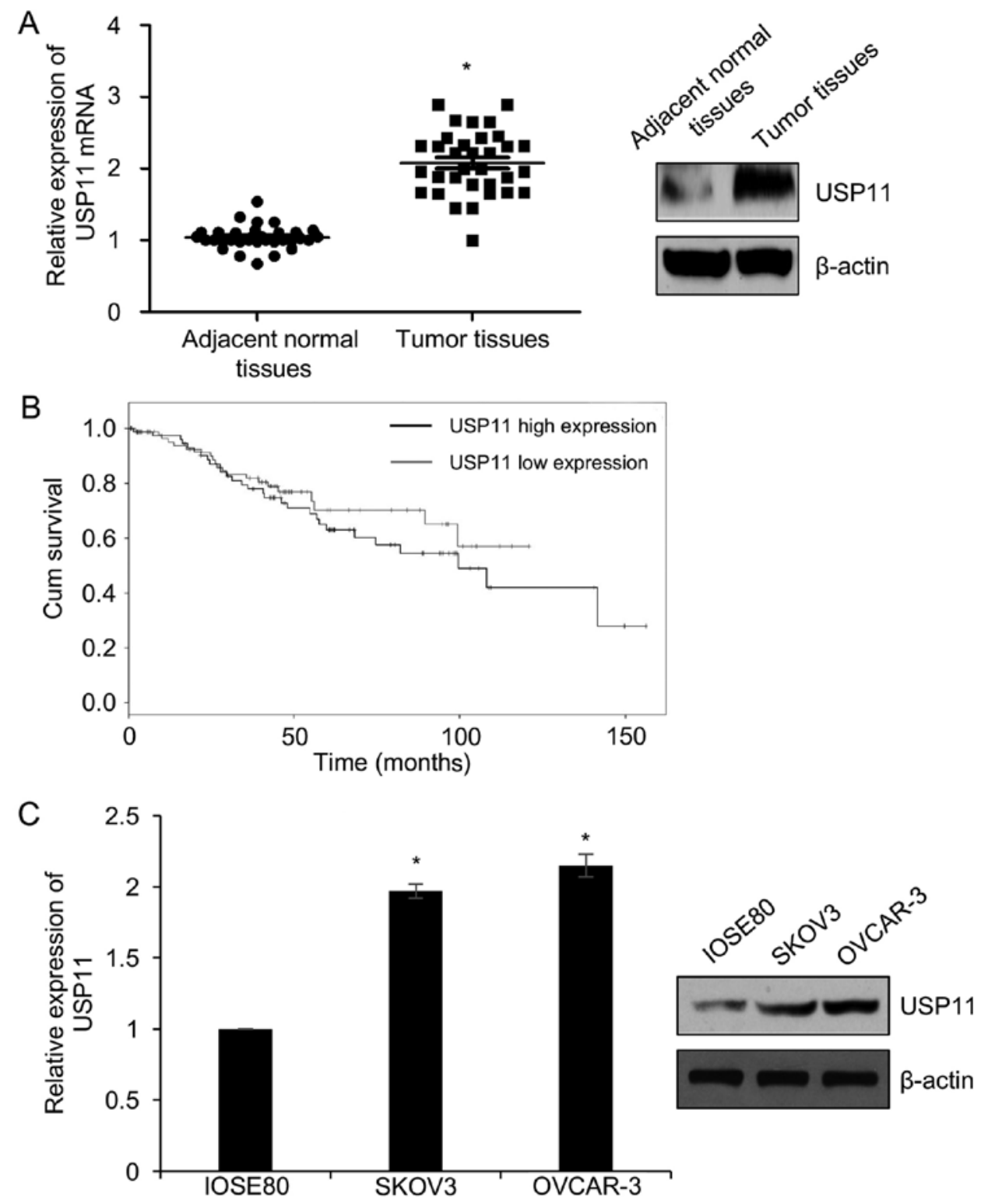

Figure 1. Association between USP11 expression and patient survival in ovarian cancer. (A) The relative expression of USP11 in tumor and adjacent normal tissues was determined by RT-qPCR and western blotting analysis. ${ }^{*} \mathrm{P}<0.05$, tumor tissues vs. adjacent normal tissues. (B) Kaplan-Meier survival analysis of the correlation between USP11 expression and overall survival (OS). (C) The relative expression of USP11 in human ovarian cancer cell lines (SKOV3 and OVCAR-3) and human normal epithelial cell line IOSE80 were determined by RT-qPCR and western blotting analysis. "P<0.05, SKOV3 or OVCAR-3 vs. IOSE80. USP11, ubiquitin specific peptidase 11.

Knockdown of USP11 suppresses the migration and invasion of ovarian cancer cell lines. Because high USP11 expression in ovarian cancer tissues was associated with lymph node metastasis, we assumed that USP11 may also regulate the migration and invasion of ovarian cancer cells. To investigate the effect of USP11 on cell migration and invasion in ovarian cancer, we first over-expressed or knocked down USP11 in SKOV3 cells and RT-qPCR and western blotting were used to determine the expression of USP11. As shown in Fig. 2A and B, USP11 was significantly over-expressed or knocked down in the SKOV3/USP11 group or the SKOV3/siUSP11 cell group, respectively. The USP11 siRNA\#1 (siUSP11\#1) showed more silencing efficiency than siRNA\#2 (siUSP11\#2); thus, siUSP11\#1 was used for further experiments. Subsequently, Transwell migration and invasion analyses were performed to determine the effect of USP11 on migration and invasion. The results of the Transwell migration assay showed that ectopic expression of USP11 increased the number of migrating cells, compared with the vector group, whereas knockdown of USP11 significantly decreased the number of migrating cells, compared with the scramble siRNA (SCR) group (Fig. 2C). Similar results were observed for the Transwell invasion assay. Ectopic expression of USP11 promoted cell invasion, compared with the vector group, whereas knockdown of USP11 significantly suppressed cell invasion, compared with the scramble siRNA (SCR) group (Fig. 2D). The above experiments suggest that USP11 promotes cell migration and invasion in ovarian cancer cells.

USP11 induces an epithelial-to-mesenchymal transition (EMT) in ovarian cancer cells. To further determine whether USP11 regulates EMT in ovarian cancer cells, we overexpressed 
A

B
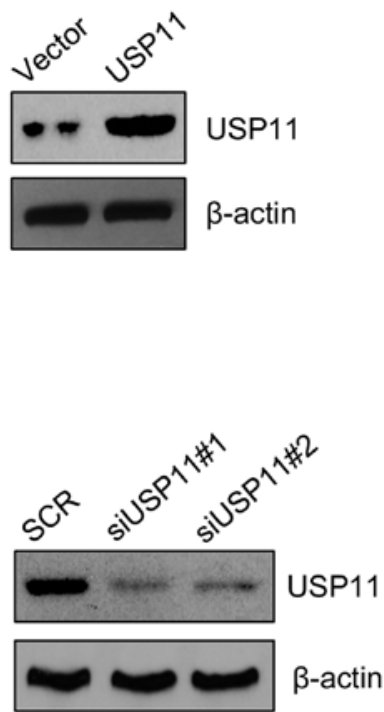

C
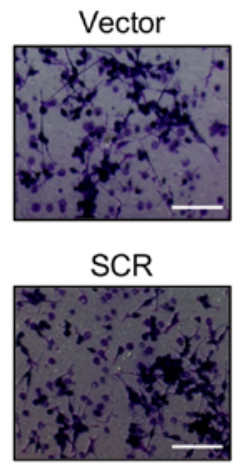

D

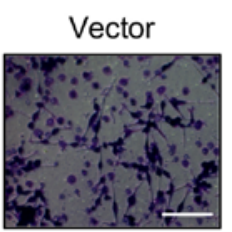

SCR

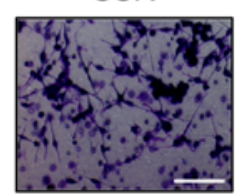

USP11

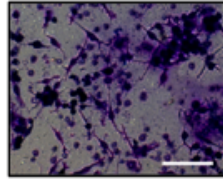

siUSP11

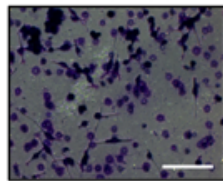

USP11

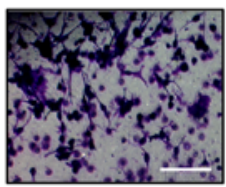

siUSP11

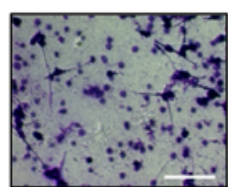

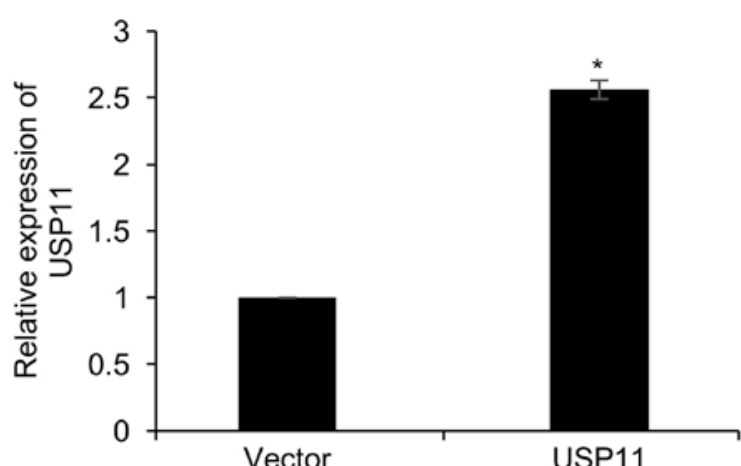
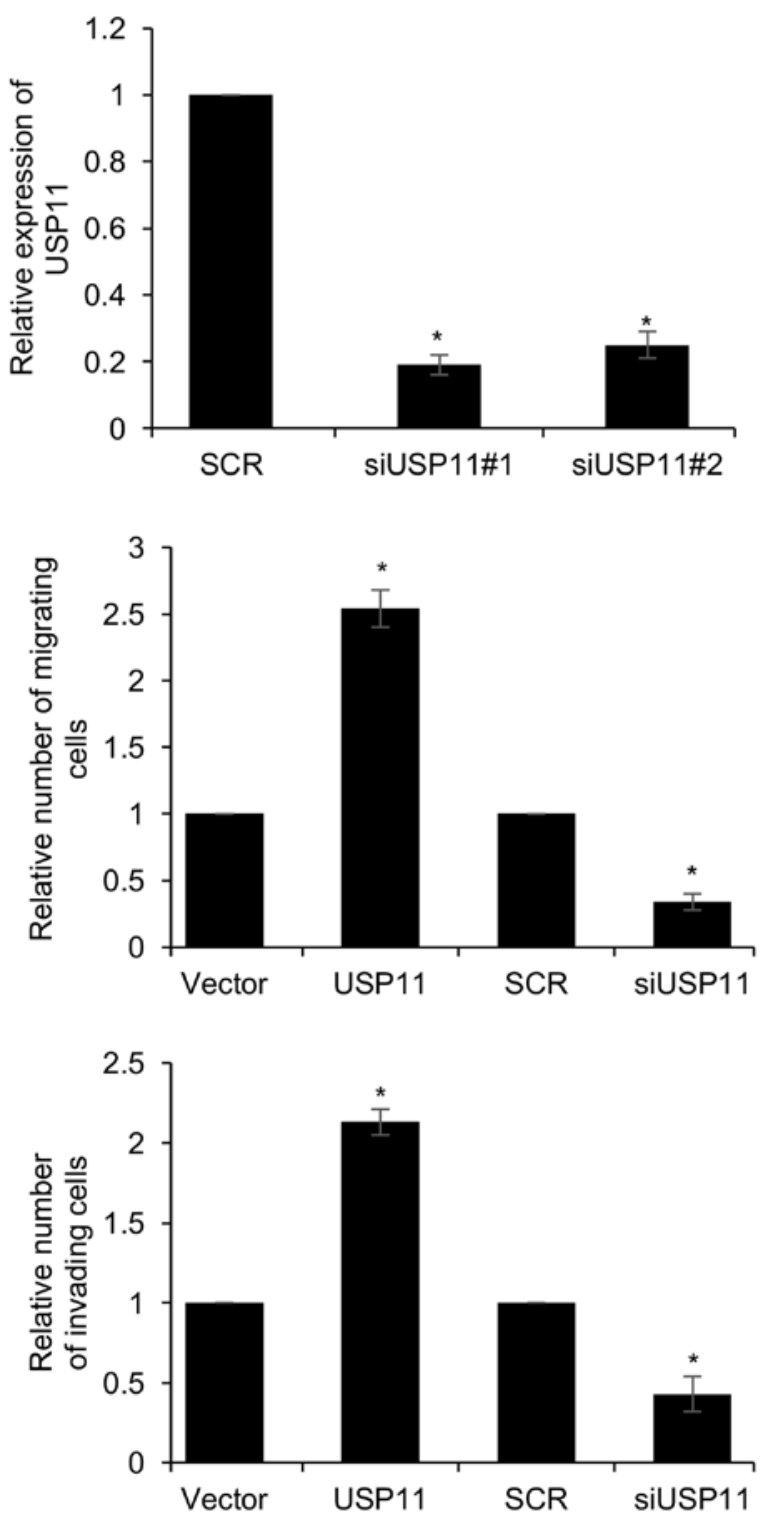

Figure 2. Knockdown of USP11 suppresses the migration and invasion of ovarian cell lines. (A) SKOV3 cells were transfected with empty vector or USP11 expression plasmid for $48 \mathrm{~h}$, and the mRNA and protein levels of USP11 were examined by RT-qPCR and western blotting. "P<0.05, USP11 vs. Vector. (B) SKOV3 cells were transfected with scramble siRNA (SCR) or USP11 siRNA\#1 (siUSP11\#1) and USP11 siRNA\#2 (siUSP11\#2) for $48 \mathrm{~h}$, and the mRNA and protein levels of USP11 were examined by RT-qPCR and western blotting. "P<0.05, siUSP11 vs. SCR. (C) USP11 was overexpressed or knocked down in SKOV3 cells. Representative images showing the migration of SKOV3 cells. The relative number of tumor cells is quantified on the right. Magnification, x20; scale bar, $100 \mu \mathrm{m}$. "P<0.05, USP11 vs. Vector, siUSP11 vs. SCR. (D) USP11 was overexpressed or knocked down in SKOV3 cells. Representative images showing the invasion of SKOV3 cells. The relative number of tumor cells is quantified on the right. Magnification, $\mathrm{x} 20$; scale bar, $100 \mu \mathrm{m}$. ${ }^{*} \mathrm{P}<0.05, \mathrm{USP} 11 \mathrm{vs}$. Vector, siUSP11 vs. SCR. USP11, ubiquitin specific peptidase 11.

or knocked down USP11 in OVCAR-3 cells, and RT-qPCR and western blotting were used to determine the expression of USP11. As shown in Fig. 3A, USP11 was significantly overexpressed or knocked down in the OVCAR-3/USP11 and 

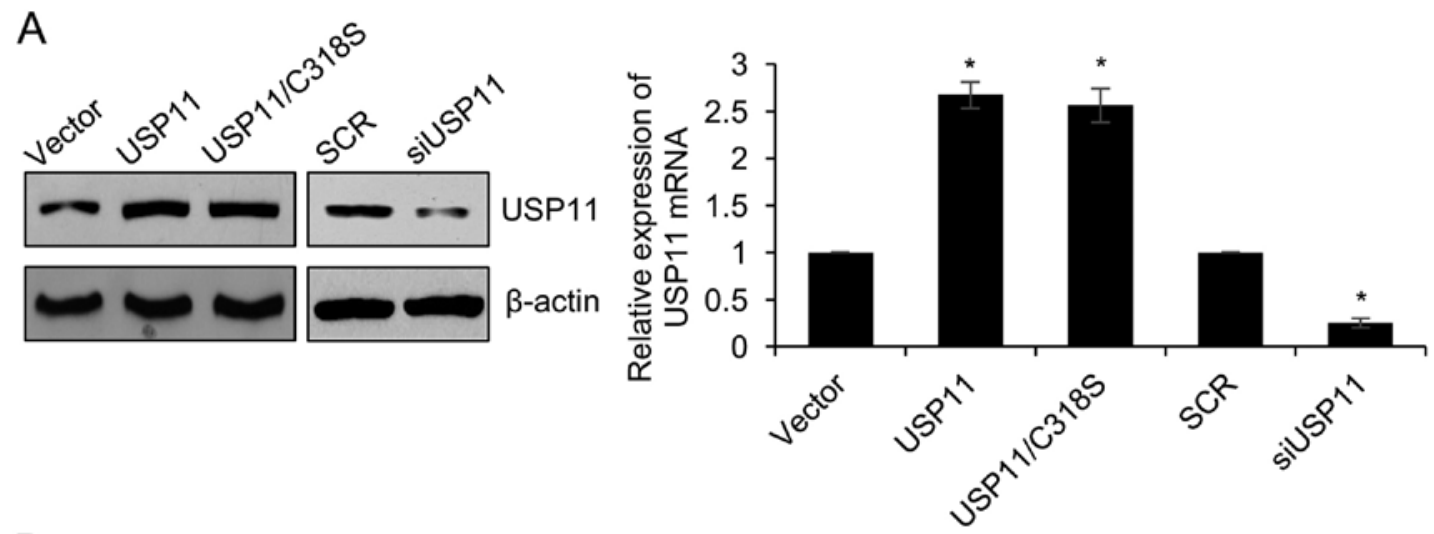

B
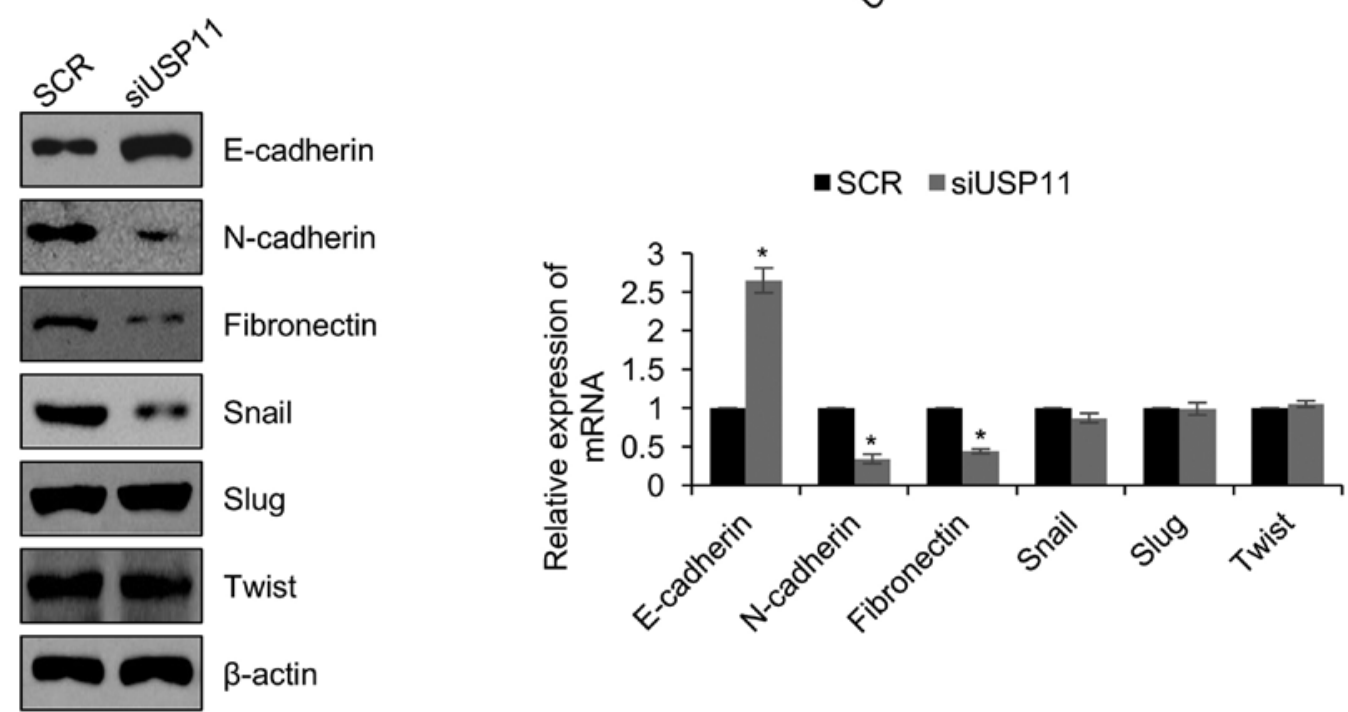

C

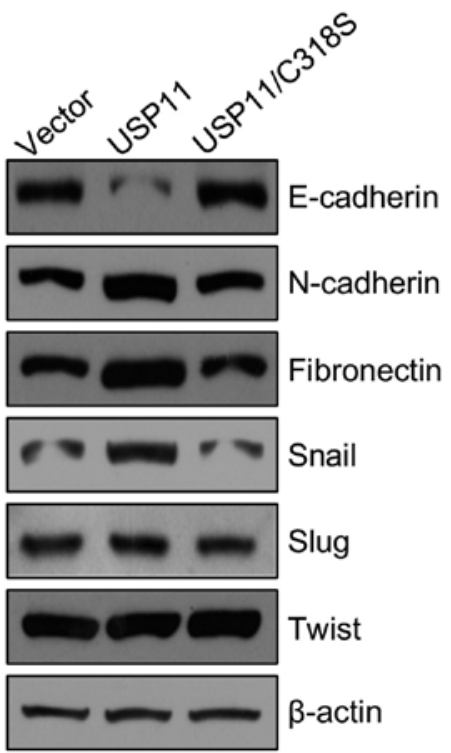

-Vector =USP11 -USP11/C318S

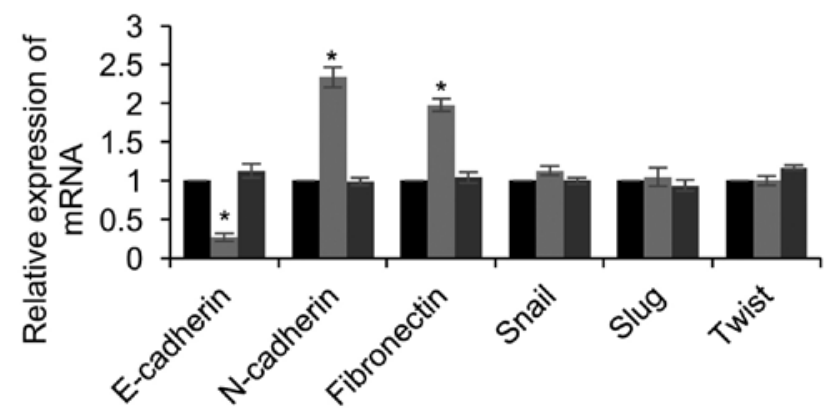

Figure 3. USP11 induces an epithelial-to-mesenchymal transition (EMT) in ovarian cancer cells. (A) USP11 was overexpressed or knocked down in OVCAR-3 cells. After transcription for $48 \mathrm{~h}$, the mRNA and protein levels of USP11 were examined by RT-qPCR and western blotting. ${ }^{\mathrm{P}}<0.05$, USP11 or USP11/C318S vs. Vector, siUSP11 vs. SCR. (B) After downregulating USP11 expression in OVCAR-3 cells, the protein and mRNA levels of EMT-associated protein were measured by western blot analysis. ${ }^{*}<0.05$, siUSP11 vs. SCR. (C) After overexpressing USP11 or USP11/C $318 S$ in OVCAR-3 cells, the protein and mRNA levels of EMT-associated proteins were measured by western blotting. " $\mathrm{P}<0.05$, USP11 or USP11/C318S vs. Vector. USP11, ubiquitin specific peptidase 11.

OVCAR-3/siUSP11 group, respectively. Next, we examined the expression of epithelial marker, E-cadherin, and the expression of the mesenchymal markers, $\mathrm{N}$-cadherin and fibronectin in OVCAR-3 cells. As shown in Fig. 3B, E-cadherin expression was increased, while $\mathrm{N}$-cadherin and fibronectin were decreased in the OVCAR-3/siUSP11 cells compared to that of the SCR control cells (Fig. 3B). Meanwhile, E-cadherin expression was decreased, while $\mathrm{N}$-cadherin and fibronectin expression were increased in the OVCAR-3/USP11 cells compared to that of the vector control cells (Fig. 3C). 
A

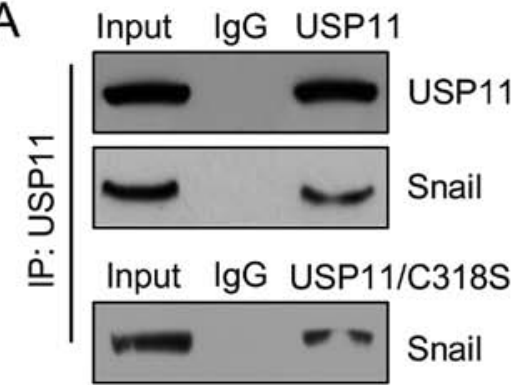

C
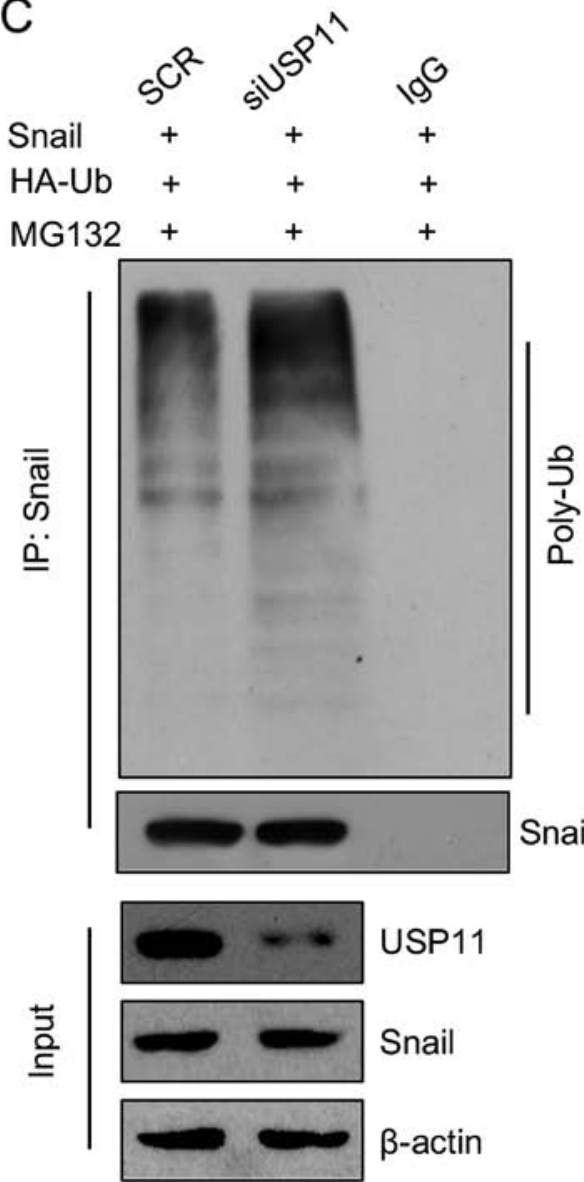

B

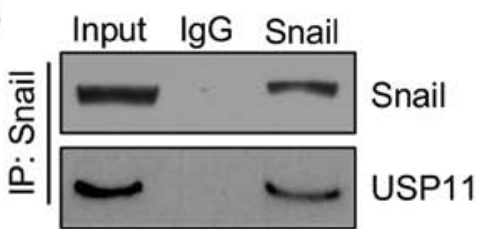

D

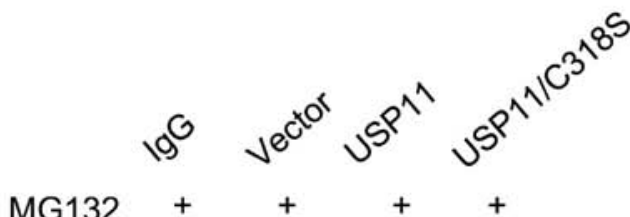

MG132 + + + + +

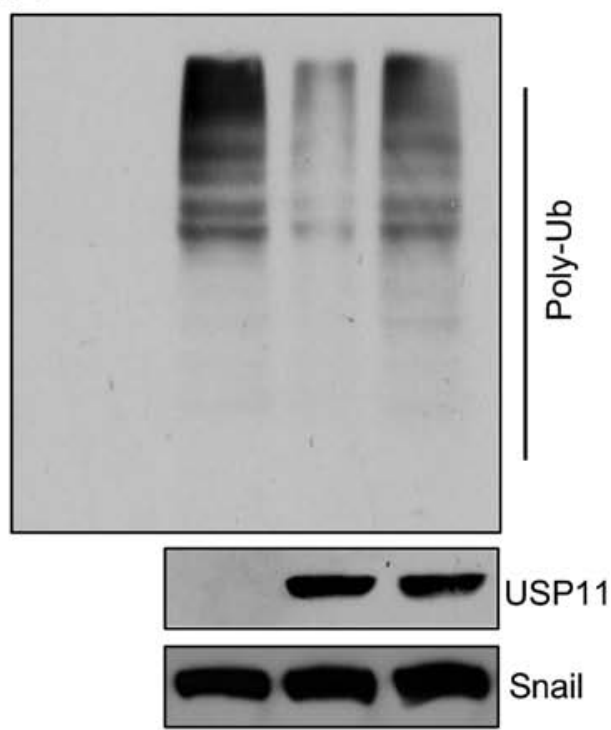

Figure 4. USP11 interacts with Snail and stabilizes Snail through its deubiquitinase activity. (A) OVCAR-3 cells were transfected with USP11 or USP11/C318S After transcription for $48 \mathrm{~h}$, the interaction between USP11 or USP11/C318S and Snail was determined by co-immunoprecipitation using anti-USP11 and anti-Snail antibodies. (B) Interaction between endogenous Snail and USP11 was determined by co-immunoprecipitation using anti-USP11 and anti-Snail antibodies. (C) USP11 was knocked down in MG132-treated OVCAR-3 cells. Extracts were subjected to IP with Snail antibody and the poly-ubiquitination (Poly-Ub) of Snail was detected by western blot analysis using anti-HA antibody. Input of USP11 and Snail were detected by western blotting. (D) Ubiquitinated Snail was purified from MG132-treated OVCAR-3 cells expressing FLAG-Snail, and then purified Snaill was incubated with purified Myc-tagged USP11 or USP11/C318S from OVCAR-3 cells expressing USP11 or USP11/C318S. The poly-ubiquitinated (Poly-Ub) state of Snail1 was determined by western blot analysis using the anti-HA antibody. USP11, ubiquitin specific peptidase 11.

To investigate whether USP11 regulates EMT through its deubiquitinase activity, we overexpressed USP11/C318S which has no catalytic activity in OVCAR-3 cells (Fig. 3A). Notably, USP11/C318S failed to regulate EMT in OVCAR-3 cells (Fig. 3C). That indicates that USP11 regulated EMT is dependent on its deubiquitinase activity. EMT-associated transcription factors, including the helix-loop-helix transcription factor Twist, and the zinc-finger containing proteins Slug and Snail, repress E-cadherin expression and induce EMT (20). To further explore the mechanism involving the regulation of EMT by USP11, we next assessed whether USP11 also regulates these transcription factors. As shown in Fig. 3B and C, the protein level of Snail was decreased when USP11 was knocked down, whereas ectopic expression of wild-type-USP11 (USP11) increased the expression of Snail at the protein level and USP11/C318S failed to increase the expression of Snail. At the mRNA level, both USP11 and USP11/C318S had no effect on the expression of Snail (Fig. 3B and C). In addition, Slug and Twist showed little change when USP11 was overexpressed or knocked down (Fig. 3B and C). The above results indicate that USP11 regulates Snail through a post-transcriptional mechanism. 

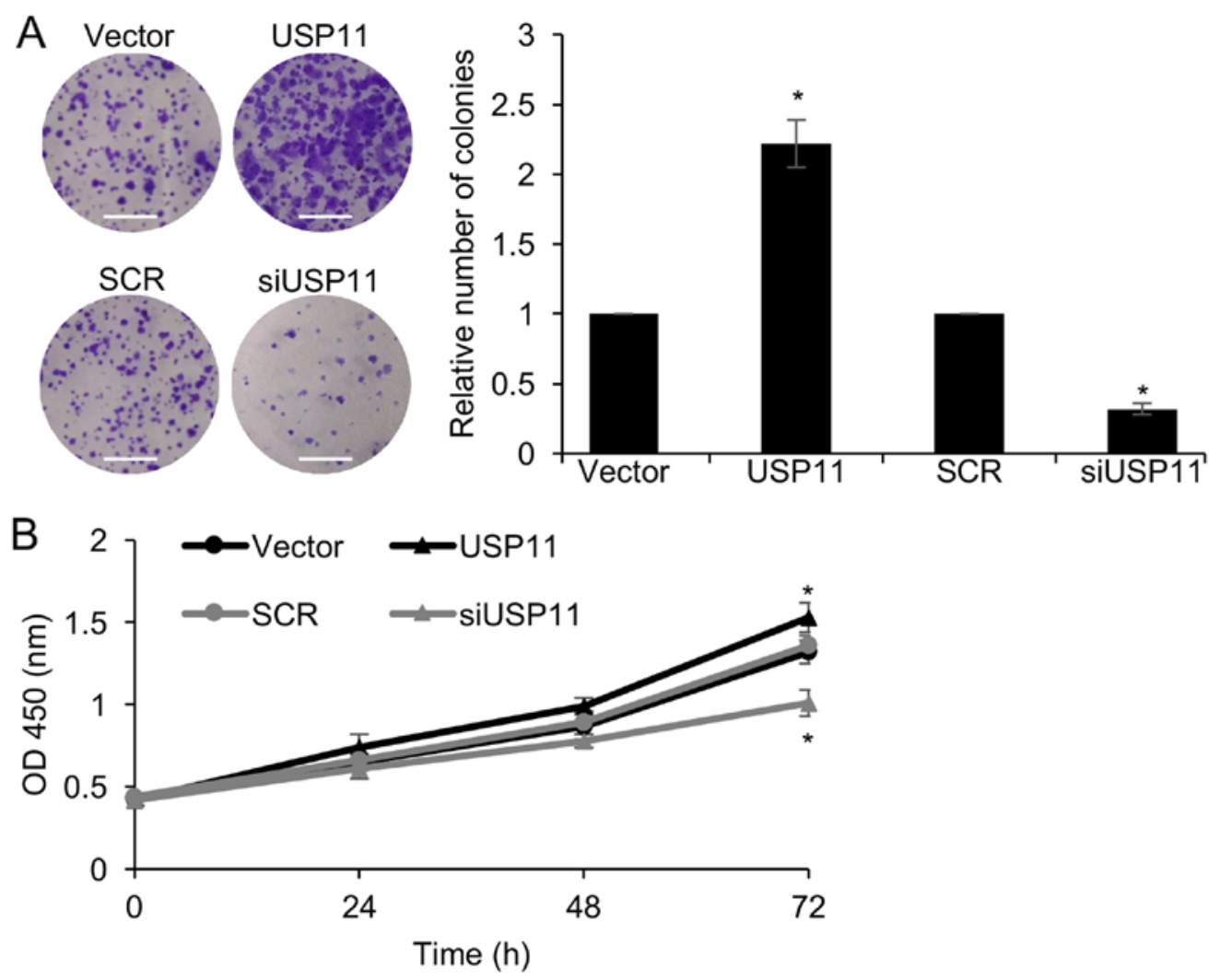

Figure 5. USP11 promotes cell proliferation in ovarian cancer cells. (A) USP11 was overexpressed or knocked down in OVCAR-3 cells, and colony formation assay was used to determine the effect of UP11 on cell proliferation. Representative images showing the number of colonies. The relative number of colonies is quantified on the right. Magnification, $\mathrm{x} 4$; scale bar, $1 \mathrm{~cm}$. "P<0.05, USP11 vs. Vector, siUSP11 vs. SCR. (B) USP11 was overexpressed or knocked down in OVCAR- 3 cells, and CCK-8 assay was used to determine the effect of UP11 on cell proliferation. ${ }^{*} \mathrm{P}<0.05$, USP11 vs. Vector, siUSP11 vs. SCR. USP11, ubiquitin specific peptidase 11.

USP11 interacts with Snail and stabilizes Snail through its deubiquitinase activity. To further decipher the detailed mechanism of USP11 on the EMT, we performed co-immunoprecipitation (co-IP) analysis to assess whether Snail interacted with USP11 or USP11/C318S. The results indicated that both USP11 and USP11/C318S could interact with Snail (Fig. 4A). Meanwhile, reciprocal immunoprecipitation with anti-Snail and immunoblotting with anti-USP11 also revealed that Snail could interact with USP11 (Fig. 4B). In brief, our research suggests USP11 is physically associated with Snail. USP11 as a deubiquitinase has been found to deubiquitinate promyelocytic leukemia protein (PML), BRCA2 and IкB $\alpha(5,8-11)$; thus, we hypothesized USP11 may regulate the expression of Snail through its deubiquitinase activity. Subsequently, we performed in vitro and in vivo deubiquitination assays. The results revealed that Snail was deubiquitinated by USP11, the ubiquitination of Snail was increased following inhibition of USP11; however, ectopic expression of USP11 decreased the ubiquitination of Snail, and USP11/C 318 S had no effect (Fig. 4C and D). Together, the above results indicate that USP11 interacts with Snail and stabilizes Snail through its deubiquitinase activity.

USP11 promotes cell proliferation in ovarian cancer cells. Since the expression of USP11 was associated with tumor size (Table I), we next explored the function of USP11 on cell proliferation. Colony formation and CCK- 8 assays were performed. The results of the colony formation assays indicated that inhibition of USP11 significantly reduced the number of colonies, compared with the SCR group; however, ectopic expression of USP11 significantly increased the number of colonies, compared with the vector group (Fig. 5A). In addition, the similar results were observed for the CCK-8 assay. Knockdown of USP11 inhibited cellular proliferation and ectopic expression of USP11 facilitated cellular proliferation (Fig. 5B). In conclusion, USP11 promotes cell proliferation in ovarian cancer cells.

\section{Discussion}

USP11 has been found to be upregulated in breast cancer, and its abnormal accumulation predicts a poor prognosis of breast cancer (6). Yet, the expression and function of USP11 in ovarian cancer remain unknown.

The results of RT-qPCR and western blotting analyses revealed that USP11 was upregulated in ovarian cancer tissues and cell lines. This finding was similar with previous research about USP11 in breast cancer (6). In addition, we also found that high expression of USP11 was closely associated with tumor size, TNM stage and lymph node metastasis. The present findings indicate that USP11 plays an important role in ovarian cancer, thus it is necessary to investigate the detailed function of USP11 in ovarian cancer.

The epithelial-to-mesenchymal transition (EMT) is a complex process that is closely related to tumor metastasis. One characteristic of EMT is the gain of mesenchymal markers, 
such as N-cadherin, and the loss of epithelial markers, such as E-cadherin. This changes the morphology of the cancer cells and improves the invasive and migratory capabilities of cancer cells. Several reports have shown that multiple transcription factors play key role in EMT, such as Snail (21-25). Abnormal expression of Snail is found in different tumor types, and its expression correlates with tumor aggressiveness $(14,26)$, and facilitates tumor recurrence (25). Here, we found that USP11 promoted the EMT in ovarian cancer cells through regulation of Snail. USP11 as a deubiquitinase has been found to interact with many proteins and stabilize them through its deubiquitinase activity. IP analysis showed that USP11 interacted with Snail and in vitro and in vivo deubiquitination assays revealed that USP11 stabilized Snail by deubiquitinating it. Meanwhile, Transwell migration and invasion assays demonstrated that USP11 enhanced the invasive and migratory capabilities of ovarian cancer cells dependent on its deubiquitinase activity.

Notably, previous research indicated that USP11 suppressed cell proliferation through regulation of Mgl-1 protein (27). However, we found the expression of USP11 was associated with a larger tumor size, and knockdown of USP11 inhibited cell proliferation in ovarian cancer cells. This suggests that USP11 may play a different role in different tumors through interacting with different proteins.

Snail has been found to be strongly regulated at the post-translational level, and it may be degraded rapidly after its initial synthesis (28). Like some other growth regulatory proteins such as cyclin D1, Myc as well as $\beta$-catenin, the degradation of Snail is associated with phosphorylation and poly-ubiquitination (28). Moreover, previous research indicates that Snail is acetylated by CREB-binding protein (CBP) (29). In particular, poly-ubiquitination can both suppress EMT by destabilizing Snail and, at the same time, enhance EMT through auto-ubiquitination of the E3 ubiquitin ligase tumor necrosis factor receptor-associated factor 6 (TRAF6) (30). Here, we found that USP11 could stabilize Snail through its deubiquitinase activity.

In conclusion, our research revealed that USP11 promoted EMT in ovarian cancer by stabilizing the EMT-associated transcription factor Snail, thereby facilitating cancer cell migration and invasion. Our results suggest USP11 as a novel molecular therapy target for ovarian cancer.

\section{Acknowledgements}

Not applicable.

\section{Funding}

No funding was received.

\section{Availability of data and materials}

The datasets used during the present study are available from the corresponding author upon reasonable request.

\section{Authors' contributions}

WW and YL conceived and designed the study. WW, JW, $\mathrm{KZ}$ and HY performed the experiments and analyzed the data. WW and $\mathrm{KZ}$ wrote the paper. WW and YL reviewed and edited the manuscript. All authors read and approved the manuscript and agree to be accountable for all aspects of the research in ensuring that the accuracy or integrity of any part of the work are appropriately investigated and resolved.

\section{Ethics approval and consent to participate}

The Ethical Committees of Linyi Central Hospital approved all human tissue experiments and all patients provided written informed consent prior to participation in the study.

\section{Patient consent for publication}

Not applicable.

\section{Competing interests}

The authors declare that they have no competing interests.

\section{References}

1. Skaar JR, Pagan JK and Pagano M: SCF ubiquitin ligase-targeted therapies. Nat Rev Drug Discov 13: 889-903, 2014.

2. Lipkowitz S and Weissman AM: RINGs of good and evil: RING finger ubiquitin ligases at the crossroads of tumour suppression and oncogenesis. Nat Rev Cancer 11: 629-643, 2011.

3. Kim D, Hong A, Park HI, Shin WH, Yoo L, Jeon SJ and Chung KC: Deubiquitinating enzyme USP22 positively regulates c-Myc stability and tumorigenic activity in mammalian and breast cancer cells. J Cell Physiol 232: 3664-3676, 2017.

4. Zhang QX, Wang XC, Chen SP and Qin XT: Predictive value of deubiquitination enzymes USP37 in the prognosis of breast cancer. Zhonghua Yi Xue Za Zhi 96: 944-948, 2016 (In Chinese).

5. Schoenfeld AR, Apgar S, Dolios G, Wang R and Aaronson SA: BRCA2 is ubiquitinated in vivo and interacts with USP11, a deubiquitinating enzyme that exhibits prosurvival function in the cellular response to DNA damage. Mol Cell Biol 24: 7444-7455, 2004.

6. Bayraktar S, Gutierrez Barrera AM, Liu D, Pusztai L, Litton J, Valero V, Hunt K, Hortobagyi GN, Wu Y, Symmans F, et al: USP-11 as a predictive and prognostic factor following neoadjuvant therapy in women with breast cancer. Cancer J 19: 10-17, 2013.

7. Kim MS, Yoo KJ, Kang I, Chung HM and Baek KH: A novel cysteine protease HeLa DUB-1 responsible for cleaving the ubiquitin in human ovarian cancer cells. Int J Oncol 25: 373-379, 2004.

8. Ramakrishna S, Suresh B and Baek KH: The role of deubiquitinating enzymes in apoptosis. Cell Mol Life Sci 68: 15-26, 2011.

9. Wu HC, Lin YC, Liu CH, Chung HC, Wang YT, Lin YW, Ma HI, Tu PH, Lawler SE and Chen RH: USP11 regulates PML stability to control Notch-induced malignancy in brain tumours. Nat Commun 5: 3214, 2014.

10. Sun W, Tan X, Shi Y, Xu G, Mao R, Gu X, Fan Y, Yu Y, Burlingame $\mathrm{S}$, Zhang $\mathrm{H}$, et al: USP11 negatively regulates TNFalpha-induced NF-kappaB activation by targeting on IkappaBalpha. Cell Signal 22: 386-394, 2010.

11. Al-Salihi MA, Herhaus L, Macartney T and Sapkota GP: USP11 augments TGFbeta signalling by deubiquitylating ALK5. Open Biol 2: 120063, 2012.

12. Gupta GP and Massague J: Cancer metastasis: Building a framework. Cell 127: 679-695, 2006.

13. Brabletz T, Kalluri R, Nieto MA and Weinberg RA: EMT in cancer. Nat Rev Cancer 18: 128-134, 2018.

14. Peinado H, Olmeda D and Cano A: Snail, Zeb and bHLH factors in tumour progression: An alliance against the epithelial phenotype? Nat Rev Cancer 7: 415-428, 2007.

15. Nagaishi M, Nakata S, Ono Y, Hirata K, Tanaka Y, Suzuki K, Yokoo $\mathrm{H}$ and Hyodo A: Tumoral and stromal expression of Slug, ZEB1, and ZEB2 in brain metastasis. J Clin Neurosci 46: 124-128, 2017. 
16. Grzegrzolka J, Biala M, Wojtyra P, Kobierzycki C, Olbromski M, Gomulkiewicz A, Piotrowska A, Rys J, Podhorska-Okolow M and Dziegiel P: Expression of EMT markers SLUG and TWIST in breast cancer. Anticancer Res 35: 3961-3968, 2015.

17. Soule HD, Vazguez J, Long A, Albert S and Brennan M: A human cell line from a pleural effusion derived from a breast carcinoma. J Natl Cancer Inst 51: 1409-1416, 1973.

18. Livak KJ and Schmittgen TD: Analysis of relative gene expression data using real-time quantitative PCR and the $2^{-\Delta \Delta C_{\mathrm{T}}}$ method. Methods 25: 402-408, 2001

19. Zhou Z, Luo A, Shrivastava I, He M, Huang Y, Bahar I, Liu Z and Wan Y: Regulation of XIAP turnover reveals a role for USP11 in promotion of tumorigenesis. EBioMedicine 15: 48-61, 2017.

20. Yang J, Mani SA, Donaher JL, Ramaswamy S, Itzykson RA, Come C, Savagner P, Gitelman I, Richardson A and Weinberg RA: Twist, a master regulator of morphogenesis, plays an essential role in tumor metastasis. Cell 117: 927-939, 2004.

21. Zheng $\mathrm{H}$ and Kang Y: Multilayer control of the EMT master regulators. Oncogene 33: 1755-1763, 2014.

22. Lamouille S, Xu J and Derynck R: Molecular mechanisms of epithelial-mesenchymal transition. Nat Rev Mol Cell Biol 15: 178-196, 2014.

23. Vetter G, Le Bechec A, Muller J, Muller A, Moes M, Yatskou M, Al Tanoury Z, Poch O, Vallar L and Friederich E: Time-resolved analysis of transcriptional events during SNAI1-triggered epithelial to mesenchymal transition. Biochem Biophys Res Commun 385: 485-491, 2009.
24. Naber HP, Drabsch Y, Snaar-Jagalska BE, ten Dijke P and van Laar T: Snail and Slug, key regulators of TGF- $\beta$-induced EMT, are sufficient for the induction of single-cell invasion. Biochem Biophys Res Commun 435: 58-63, 2013.

25. Moody SE, Perez D, Pan TC, Sarkisian CJ, Portocarrero CP, Sterner CJ, Notorfrancesco KL, Cardiff RD and Chodosh LA: The transcriptional repressor Snail promotes mammary tumor recurrence. Cancer Cell 8: 197-209, 2005.

26. Blanco MJ, Moreno-Bueno G, Sarrio D, Locascio A, Cano A, Palacios J and Nieto MA: Correlation of snail expression with histological grade and lymph node status in breast carcinomas. Oncogene 21: 3241-3246, 2002.

27. Lim KH, Suresh B, Park JH, Kim YS, Ramakrishna S and Baek KH: Ubiquitin-specific protease 11 functions as a tumor suppressor by modulating Mgl-1 protein to regulate cancer cell growth. Oncotarget 7: 14441-14457, 2016.

28. Zhou BP, Deng J, Xia W, Xu J, Li YM, Gunduz M and Hung MC: Dual regulation of Snail by GSK-3beta-mediated phosphorylation in control of epithelial-mesenchymal transition. Nat Cell Biol 6: 931-940, 2004.

29. Hsu DS, Wang HJ, Tai SK, Chou CH, Hsieh CH, Chiu PH, Chen NJ and Yang $\mathrm{MH}$ : Acetylation of snail modulates the cytokinome of cancer cells to enhance the recruitment of macrophages. Cancer Cell 26: 534-548, 2014.

30. Gudey SK,SundarR,Mu Y,Wallenius A,Zang G,BerghA,HeldinCH and Landström M: TRAF6 stimulates the tumor-promoting effects of TGF $\beta$ type I receptor through polyubiquitination and activation of presenilin 1. Sci Signal 7: ra2, 2014. 